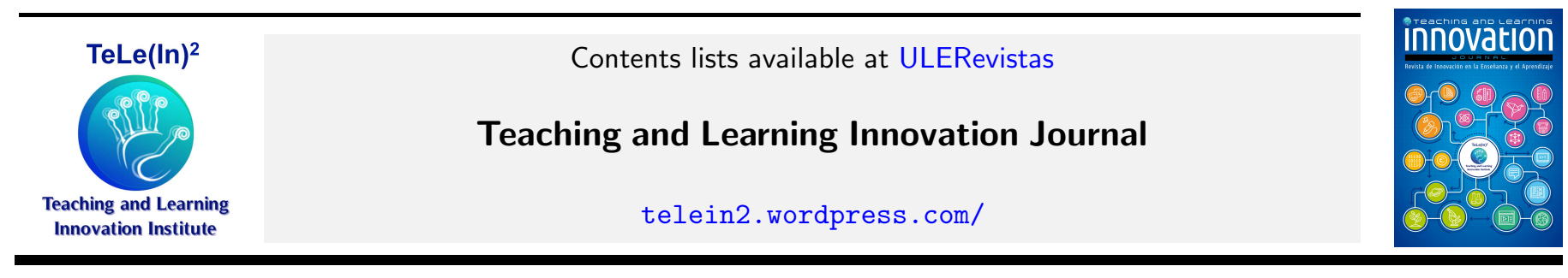

\title{
Incorporación de la metodología "Service-learning" en la Facultad de Ciencias Biológicas y Ambientales de la Universidad de León \\ Introduction of Service-learning teaching method at the Faculty of Biology and Environmental Science (University of Leon)
} \author{
Aller $^{\mathrm{d}}$, R.M. Valencia Barrera ${ }^{\mathrm{a}}$, L.F. Valladares Díez ${ }^{\mathrm{a}}$, M.P. Valle Fernández ${ }^{\mathrm{d}}$, B. Razquin Peralta ${ }^{\mathrm{d}}$

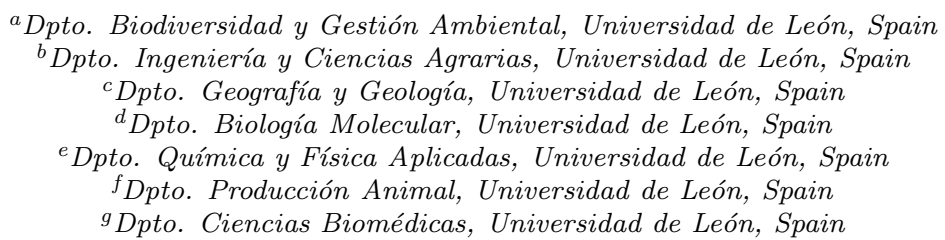

L. Calvo Galván ${ }^{\mathrm{a}}$, M.L. Centeno Martín ${ }^{\mathrm{b}}$, E. Colmenero Hidalgo ${ }^{\mathrm{c}}$, P. García García ${ }^{\mathrm{d}}$, A. Fernández Villadangos ${ }^{\mathrm{d}}$, L. López Campano $^{\mathrm{e}}$, M. Marqués Martínez ${ }^{\mathrm{f}}$, C. Marín Vieira ${ }^{\mathrm{d}}$, L.M. Mateos Delgado ${ }^{\mathrm{d}}$, J.L. Máuriz Gutiérrezº ${ }^{\mathrm{g}}$ F.J. Rúa

\begin{abstract}
La metodología docente "service-learning", como su nombre indica, combina en un proyecto único, un servicio a la comunidad con un aprendizaje académico relacionado con el curriculum del estudiante. En esta comunicación se presentan los resultados obtenidos en dos proyectos docentes que han utilizado este método para facilitar a los estudiantes de Grado la adquisición de conocimientos significativos y el desarrollo de habilidades personales y sociales difíciles de conseguir con las metodologías tradicionales. Estos proyectos han sido desarrollados por un grupo de profesores que imparten docencia en la Facultad de Ciencias Biológicas y Ambientales de la Universidad de León, en los Grados en Biología, Biotecnología y Ciencias Ambientales, durante los cursos 2014-15 y 2015-16. Con la introducción de la metodología docente "service-learning" se consigue que la Universidad se abra a la sociedad y alcance una conexión real con su entorno, avanzando en lo que se ha denominado la tercera misión de la universidad. El fin último de estos proyectos, es que tanto los estudiantes como los profesores universitarios conozcan las potencialidades de esta metodología y se planteen incorporarla institucionalmente, como ya se hace en otras Universidades americanas y europeas.
\end{abstract}

Service-learning is a teaching and learning methodology that integrates community service with academic coursework, enhancing the students' learning experience. This communication summarizes the results obtained in two teaching projects in which students applied course contents to community-based activities, incorporating learning and community action goals. These projects were designed by a group of university professors from the Faculty of Biological and Environmental Sciences of the University of León, and were carried out by a selected group of students from the Degrees in Biology, Biotechnology and Environmental Sciences, in collaboration with different community partners. The purpose of these teaching experiences was to provide the students with a hand-on experience that linked personal and social development, helping them to develop new skills difficult to acquire with traditional teaching methods. Moreover, service-learning projects enhance community-university relations improving institutional commitment with the society. Our final goal is to publicize the benefits of community engagement pedagogies like service-learning among students and professors and to set the path to integrate them into the existing curriculum of the University of Leon.

Keywords: Aprendizaje-servicio, adquisición de competencias, Ciencias. service-learning, skills acquisition, Science.

Email addresses: leonor.calvo@unileon.es (L. Calvo Galván), mlcenm@unileon.es (M.L. Centeno Martín), e.colmenero@unileon.es (E. Colmenero Hidalgo), pgarg@unileon.es (P. García García), aferv@unileon.es (A. Fernández Villadangos), laura.lopez@unileon.es (L. López Campano), mmarm@unileon.es (M. Marqués Martínez), 


\section{Introducción}

La sociedad actual exige que las enseñanzas universitarias no se centren únicamente en la simple transmisión de conocimientos, sino que además, consigan desarrollar en los estudiantes las competencias profesionales, sociales y éticas que les permitan hacer frente en las mejores condiciones a las nuevas situaciones que van a vivir. Este tipo de aprendizaje es difícil de conseguir si seguimos basando nuestras enseñanzas exclusivamente en las metodologías clásicas.

El método docente "service-learning" es una propuesta educativa que combina procesos de aprendizaje y de servicio a la comunidad en un proyecto único (Honnet EP, Poulson SJ , 1989, Arroyes, A. et al., 2010). Al unir estos dos elementos consigue, por un lado, que el aprendizaje adquiera sentido, es decir, el estudiante se da cuenta de que lo que está aprendiendo es útil, y por otro lado, se desarrolla un servicio solidario. La metodología del "servicelearning" promueve que los estudiantes salgan del mundo académico, se enfrenten a problemas reales y apliquen los conocimientos adquiridos para solucionarlos. Por ello, se considera una forma eficaz de llevar a cabo algunos de los cambios docentes que la Universidad debe realizar.

El "service-learning" empezó a desarrollarse en Estados Unidos a partir de los ochenta y posteriormente su uso se extendió por Sudamérica durante la década de los 90 (Bringle, R.G. \& Hatcher, J.A., 1996). En Europa, y sobre todo en España, el desarrollo es más reciente. Actualmente, existen experiencias de incorporación de esta metodología en algunas universidades españolas, como la del País Vasco, la Pública de Navarra, la Autónoma de Barcelona, la Autónoma de Madrid, algunas universidades andaluzas, etc., como se refleja en la Red española de aprendizaje-servicio (5) y en la Red universitaria de aprendiza servicio (6). Las experiencias realizadas (Martínez, B., Martínez, Abes, E. et al. 2002. F I., Folgueiras, P. et al, 2013, Alonso, I. y Gezuraga, M., 2013 y García, M.R. \& Green, C.G., 2008) muestran que el "service-learning" es una metodología que facilita la adquisición de competencias profesionales y personales de los estudiantes, además de favorecer la colaboración entre docentes y cambiar la visión que tiene la comunidad de la Universidad.

\section{Contexto}

El objetivo de estos proyectos ha sido la incorporación de esta metodología en las enseñanzas de la Facultad de Ciencias Biológicas y Ambientales (FCCBA) de la Universidad de León (ULE) con un doble propósito. Por un

carmen.marin@unileon.es (C. Marín Vieira),

luis.mateos@unileon.es (L.M. Mateos Delgado), jl.mauriz@unileon.es (J.L. Máuriz Gutiérrez),

javier.rua@unileon.es (F.J. Rúa Aller), rm.valencia@unileon.es

(R.M. Valencia Barrera), luis-felipe.valladares@unileon.es

(L.F. Valladares Díez), mpvalf@unileon.es (M.P. Valle Fernández), blanca.razquin@unileon.es (B. Razquin Peralta) lado, como procedimiento para garantizar que los estudiantes adquieren las competencias transversales de trabajo en equipo, comunicación y transmisión de conocimientos, entre otras, que demandan los nuevos tiempos. Por otro, realizando una divulgación científica de calidad y colaborando en el fomento de las vocaciones científicas.

El primero de los proyectos, denominado "Los retos de la ciencia contados por futuros científicos" se desarrolló en el curso 2014-15 y consistió en la preparación de diez charlas divulgativas con información accesible y contrastada sobre temas científicos de actualidad que se ofertaron a diez Ayuntamientos pequeños de la provincia de León. En la Tabla 1 figura la relación de charlas divulgativas preparadas.

En la experiencia participaron diez profesores de diferentes Áreas de Conocimiento que imparten docencia en la FCCBA y se ofertó para veinte estudiantes de cuarto curso de los Grados de Biología, Biotecnología y Ciencias Ambientales. Cada profesor seleccionó un tema de actualidad de su ámbito de conocimiento y actuó como tutor de dos estudiantes a los que guio a lo largo de toda la experiencia. El trabajo de los estudiantes consistió en buscar información relevante sobre el tema seleccionado, estudiarla, sintetizarla y preparar una charla divulgativa que presentaron, atendiendo a las dudas y preguntas de los asistentes. Este proyecto permitió que los estudiantes pudiesen utilizar conocimientos y competencias adquiridas en su Grado para prestar un servicio a la comunidad, divulgando el conocimiento científico y acercando, de esta forma, a la Universidad a nuestra sociedad.

El segundo de los proyectos titulado "Viaje por la ciencia guiado por futuros científicos" se realizó en el curso 2015-16 y consistió en la organización de un programa de doce sesiones prácticas dirigidas a estudiantes je đe primero de bachillerato que se ofertó a catorce centros de enseñanza de la provincia de León. En la Tabla 2 figura la relación de sesiones prácticas preparadas. En esta experiencia participaron quince profesores de diferentes Áreas de Conocimiento, y se ofertó para veinticuatro estudiantes de los cursos tercero y cuarto de los Grados de Biología, Biotecnología y Ciencias Ambientales. Cada profesor seleccionó una práctica de su campo de conocimiento y, como en el caso anterior, actuó como tutor de dos estudiantes. Los estudiantes prepararon, organizaron y desarrollaron la práctica correspondiente en los laboratorios de la FCCBA, atendiendo además a las cuestiones planteadas por los alumnos de primero de bachillerato. En este caso, los alumnos aprendieron a enseñar a otros más jóvenes y a estimular su interés científico. Es importante resaltar que esta actividad sirvió a su vez de herramienta de presentación de la FCCBA en nuestro entorno social, y en especial, a potenciales alumnos.

Ambos proyectos han servido para dar a conocer las posibilidades de este método docente a los estudiantes y a los profesores de la Facultad y de la Universidad, con el fin de que en un futuro las actividades de este tipo puedan incluirse en los Grados universitarios. 
Tabla 1: Relación de charlas divulgativas

Los incendios forestales: un problema antiguo y un reto para el siglo XXI

Revolución en la granja: nuestras plantas y animales se alían con la Biotecnología

Nuestra herencia: viaje al interior del genoma humano

Riesgos meteorológicos en Castilla y León: predicción, seguimiento en tiempo real e influencia del cambio climático

Entendiendo el cáncer: una mirada hacia el futuro

¿Es útil el uso de ciertos seres vivos para la reparación de aguas y suelos contaminados?

Bases para un envejecimiento saludable

¿Será posible construir órganos para trasplantes con células madre?

Las invasiones biológicas, una consecuencia del cambio global

\section{Descripción}

El desarrollo de estos proyectos puede dividirse en tres etapas: la primera de planificación, una segunda de ejecución y finalmente la valoración de la experiencia y el cierre del proyecto.

\section{A. Planificación del proyecto}

En esta etapa participan los profesores implicados en la experiencia e incluye: a) la elección de los temas de trabajo; b) la elaboración del calendario de realización de las actividades; c) la valoración de las horas de trabajo de los estudiantes de Grado participantes; d) la solicitud al Vicerrectorado correspondiente del reconocimiento de 3 ECTS para los estudiantes de Grado; e) La presentación y explicación del proyecto a los estudiantes de los cursos tercero y cuarto de Grado; f) la selección de los estudiantes; y g) el establecimiento de contactos con las entidades, ayuntamientos y Centros de Enseñanza respectivamente a los que se ofertan las actividades.

\section{B. Ejecución del proyecto}

En esta etapa participan los profesores y los estudiantes de Grado seleccionados y se realiza en un horario consensuado entre todos para que no interfiera con las actividades docentes del semestre.

Los estudiantes, orientados por sus profesores-tutores, llevaron a cabo un trabajo autónomo en el que consultaron la bibliografía, planificaron la charla o la sesión práctica y prepararon una presentación. Además, desarrollaron un trabajo presencial con sus tutores durante el cual discutieron la bibliografía seleccionada, resolvieron sus dudas, se revisaron y corrigieron las presentaciones y se realizaron ensayos previos de la actividad. Paralelamente, los profesores elaboraron los cuestionarios para la evaluación de la actividad y se diseñaron e imprimieron los carteles anunciadores de las actividades.
Tabla 2: Relación de sesiones prácticas en laboratorio

Estudio de células animales y vegetales con el microscopio óptico

¿Cuánto almidón hay en los alimentos? Aislamiento e hidrólisis

Adaptaciones de las plantas a la polinización

Producción primaria en ecosistemas terrestres

Aplicaciones de la termografía infrarroja

Estudio de las células sanguíneas: eritrocitos y leucocitos Conociendo cómo funcionan las plantas

¿Por qué no nos gustan los mismos alimentos a todos? Genética de la percepción de los sabores

ATENCIÓN, nos rodean los microorganismos

Biotecnología y producción de leche: aplicaciones a pie de campo

Los materiales de la corteza terrestre: identificación y observación de minerales y rocas

Morfología y taxonomía prácticas: los crustáceos como modelo

Finalmente, los estudiantes, siempre acompañados por los profesores, presentaron las charlas y desarrollaron las prácticas correspondientes y respondieron a las dudas planteadas por los asistentes.

\subsection{Valoración y cierre del proyecto}

Esta etapa incluye la evaluación de la experiencia realizada y la elaboración de una memoria final.

La evaluación se basó en las opiniones de: a) los asistentes a las charlas o a las sesiones prácticas, que mediante la encuesta que se distribuyó al final de cada una de la actividades valoraron el interés y la utilidad de los temas tratados, el trabajo de las personas que las impartieron y la conveniencia de este tipo de actividades; b) los estudiantes de Grado que participaron, los cuales a través de la encuesta que se les pasó y del informe final que presentaron valoraron el trabajo realizado, los conocimientos y las competencias adquiridas y los beneficios y dificultades de la experiencia; c) los profesores participantes que, por medio también de una encuesta y de un informe final, valoraron el trabajo que había supuesto para ellos la realización del proyecto, los resultados obtenidos y las posibilidades docentes del "service-learning".

Con todos los datos recogidos se elaboró la memoria final del proyecto.

\section{RESULTADOS}

Proyecto "Los retos de la ciencia contados por futuros cientificos"

En este proyecto participaron 20 estudiantes, de los cuales 12 eran del Grado en Biología, 3 del Grado en Biotecnología y 5 del Grado en Ciencias Ambientales. Al 
finalizar la experiencia, todos ellos manifestaron su satisfacción personal por haber participado en un proyecto de este tipo y destacaron la ayuda recibida de los profesores tutores, los momentos agradables del trabajo en equipo y la soltura y seguridad adquiridas para hablar ante un público y en un lugar desconocido. Como aspecto negativo, resaltaron la falta de tiempo para trabajar desahogadamente. En todos los casos, indicaban que recomendarían a otros estudiantes la participación en este tipo de actividades.

A las charlas divulgativas asistieron 323 personas, de edades comprendidas entre los 45 y los 60 años. La mayor parte de ellos consideraron las charlas muy interesantes y calificaron la exposición como clara, amena y divulgativa (Figura 1). Valoraron muy positivamente este tipo de actividades y el acercamiento de la Universidad a la sociedad. Este último aspecto era más evidente cuando se leían los comentarios elogiosos que incluían las encuestas, felicitando a la Concejalía de Cultura por haber acogido la experiencia y pidiendo que se repitiera en ediciones sucesivas.

Para los profesores la experiencia resultó también satisfactoria y resaltaron como aspectos positivos, la implicación de los estudiantes de Grado y el establecimiento de contactos entre la Universidad y los pueblos del entorno. Aunque también manifestaron que la carga de trabajo había sido mayor de lo esperado, y la enorme dificultad para encontrar tiempo disponible para trabajar con los estudiantes dada la complejidad y extensión de los horarios docentes.

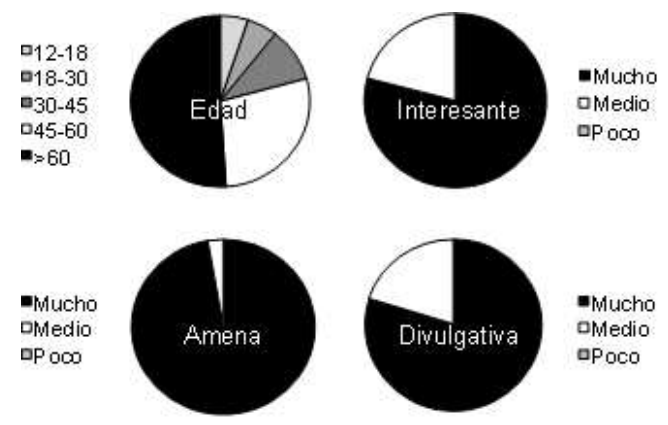

Figure 1: Edad y opinión de los asistentes a las charlas divulgativas.

\subsection{B. Proyecto "Viaje por la ciencia guiado por futuros científicos"}

En este segundo proyecto participaron 24 estudiantes, de los cuales 17 eran del Grado en Biología, 5 del Grado en Ciencias Ambientales y 2 del Grado en Biotecnología. En los informes y en las encuestas de valoración que presentaron al finalizar la experiencia, destacaron la atención y el asesoramiento recibido por parte de los profesorestutores, así como el aprendizaje que les había supuesto ponerse en el papel de profesor y comprender el trabajo previo que implica la preparación de una clase práctica. Además, consideraron que habían mejorado su capacidad de trabajar en equipo, manejo de la información y facilidad de hablar en público. Todos ellos indicaron que, a pesar de la falta de tiempo, la actividad les resultó muy grata, les pareció un buen complemento a su formación, y recomendarían a otros compañeros que participasen en este tipo de actividades.

A las sesiones prácticas organizadas asistieron 215 estudiantes de primero de Bachillerato de 14 centros tanto de la ciudad de León, como de otras localidades de la provincia (Figura 2). Incluso hubo que excluir de este proyecto algunos Centros debido a la limitación temporal y la capacidad de que disponíamos.

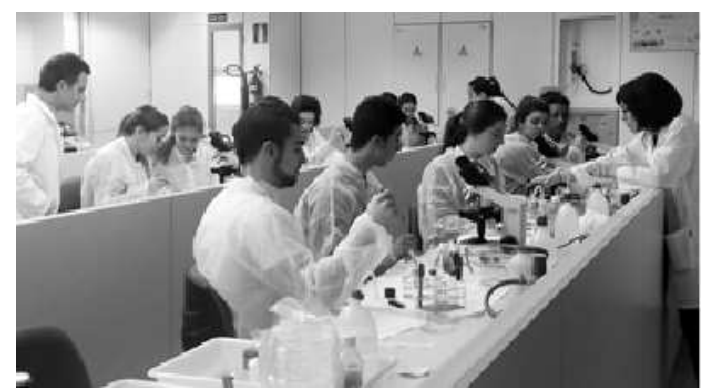

Figure 2: Sesión práctica "ATENCIÓN, nos rodean los microorganismos".

Los profesores de los Institutos, que acompañaron a los estudiantes y asistieron con ellos a las sesiones prácticas, valoraron muy positivamente tanto la organización como el desarrollo de las sesiones prácticas. Les pareció muy buena idea esta colaboración de la Universidad con los Centros de Secundaria y solicitaron que se repitiera la experiencia en años sucesivos. En cuanto a la opinión de los estudiantes de bachillerato, manifestada en las encuestas que se pasaron al finalizar las sesiones prácticas, fue en todos los casos muy favorable, las prácticas les resultaron muy interesantes, pudieron seguirlas sin dificultad y valoraron muy bien la labor de los profesores y el esfuerzo de los estudiantes que se las impartieron. Todos indicaron que repetirían la experiencia.

También para los profesores tutores la actividad resultó satisfactoria y destacaron la disponibilidad e implicación de los estudiantes. Todos valoraron positivamente la realización de este tipo de actividades que acercan la Universidad a los estudiantes de secundaria y bachillerato y consideran que experiencias de este tipo deberían repetirse de manera regular como estrategia de la ULE para darse a conocer en su entorno.

\section{Conclusiones}

Como se desprende de los resultados expuestos en el apartado anterior, el desarrollo de estos proyectos ha de- 
mostrado que la metodología "service-learning": a) aporta beneficios tanto para los estudiantes y los docentes como para la propia Universidad y la sociedad del entorno. A los estudiantes les ayuda a adquirir competencias relevantes para su futuro profesional, mientras que a los profesores les permite sacar la enseñanza fuera del aula y establecer contactos con organizaciones y personas que no pertenecen al ámbito académico universitario. En cuanto a la comunidad, destinataria de estas actividades, recibe un servicio gratuito de calidad y mejora su visión de la Universidad, al percibirla como un ente más cercano y útil. Finalmente, se facilita el establecimiento de redes de colaboración entre la Universidad y otros agentes de la sociedad; b) puede incluir un amplio campo de proyectos, tanto de la rama de Ciencias como de cualquier otra de la universidad. Por lo tanto, es fácilmente transferible a otras titulaciones universitarias. Así, lo demuestran las experiencias de las universidades en las que se ha implantado; c) puede representar un eje de actuación para aumentar la visibilidad y el acercamiento de la Universidad a la sociedad leonesa y para captar nuevos estudiantes universitarios.

Sin embargo, la organización de proyectos de este tipo es compleja y conlleva mucho tiempo tanto para los profesores como para los estudiantes. Por eso, sería conveniente que las actividades de este tipo fueran impulsadas por las autoridades académicas, se incluyeran en los planes de estudio de los Grados, por ejemplo, en forma de asignaturas optativas y contaran con un apoyo institucional.

\section{Agradecimientos}

Agradecemos a la Escuela de Formación de la ULE que ha financiado la realización de estos proyectos y a la FCCBA que ha puesto a nuestra disposición las infraestructuras necesarias y nos ha facilitado la realización de las actividades. También queremos agradecer a los estudiantes de Grado que han participado en estas experiencias su implicación entusiasta a las mismas.

\section{Referencias}

[1] Abes, E. et al. (2002). Factors that Motivate and Deter Faculty Use of Service-Learning, Michigan Journal of Community Service Learning, 9: 5-17.

[2] Arroyes, A. et al. (2010). Prácticas de ciudadanía: diez experiencias de Aprendizaje Servicio. Barcelona: Ediciones Octaedro.

[3] Bringle, R.G. \& Hatcher, J.A. (1996). Implementing service learning in higher education. Journal of higher education 67 : 221- 239.

[4] Folgueiras, P. et al. (2013). Aprendizaje y servicio: estudio del grado de satisfacción de estudiantes universitarios. Revista de Educación 362: 159-185.

[5] Honnet EP, Poulson SJ. Principles of good practice for combining service and learning (Wingspread Report) Racine Wis: The Johnson Foundation, Inc; 1989.

[6] Martínez, B., Martínez, I., Alonso, I. y Gezuraga, M. (2013). El Aprendizaje-Servicio, una oportunidad para avanzar en la innovación educativa dentro de la Universidad del País Vasco. Tendencias Pedagógicas 21: 99-117
[7] García, M.R. \& Green, C.G. (2008). El "Service-Learning" o Aprendizaje-Servicio como método de enseñanza: experiencia de la Pace University (Nueva York) en la creación de "green maps" o mapas verdes en el marco del desarrollo sostenible del turismo. Revista de enseñanza universitaria 32: 13-28.

[8] Red española de Aprendizaje-Servicio http://aprendizajeservicio.net/index.html

[9] Red universitaria española de Aprendizaje-Servicio https://sites.google.com/site/redapsuniversitario/home 